Methodology Patients treated with PARPis between November 2016 and December 2020 were enrolled in this prospective study. PRO-CTCAE questionnaires were generated on the specific toxicities of PARPis using the form builder developed by the Division of Cancer Control and Population Science in the National Cancer Institute at the National Institute of Health and administered to the cohort. Patients toxicities, as recorded by physicians, were analyzed and compared with monthly PRO-CTAE questionnaires.

Result(s)* Thirty-one EOC patients underwent maintenance therapy with PARPis after 1 (24\%), 2 (48\%) and $\geq 3(28 \%)$ lines of chemotherapy. The median age was 56 (range 35-77), 83.3\% of patients had an ECOG Performance Status 0 and $14(45.2 \%)$ were BRCA mutated. 50\% received olaparib, $42.9 \%$ niraparib and 7,1\% rucaparib. No patient discontinued treatment due to toxicity and $38.7 \%$ delayed the treatment due to anaemia (29\%) or thrombocytopenia $(9,7 \%)$. Haematological toxicities and asthenia were the most frequent adverse events recorded by physicians and occurred in $42.5 \%$ and $45.2 \%$ of patients, respectively. Concordance between the toxicity reported by patients and by physicians was observed in $40 \%$ of cases. PRO-CTCAE questionnaires contributed to the toxicity evaluation revealing symptoms under-reported by physicians, in particular: $35.7 \%$ of anorexia, $79.5 \%$ of nausea, $90 \%$ of vomiting, $63.7 \%$ of constipation, $79.8 \%$ of diarrhea, $35.3 \%$ of asthenia, $87.4 \%$ of arthralgia and $100 \%$ of headache and insomnia.

Conclusion* PRO-CTCAE is a toxicity assessment tool that should be required especially in the monitoring of maintenance treatments. The physician's evaluation of toxicities, enriched by the patient reported outcomes, could allow more targeted and earlier interventions and potentially affect the adherence to the treatment.

All authors have no conflict of interest

\section{MULTIMODAL SCORING SYSTEM FOR RO RESECTION IN OVARIAN CANCER}

1,2F Saner*, ${ }^{3} \mathrm{~K}$ Härmä, 'S Imboden, 'MD Mueller. 'Univeristy Hospital Bern, Inselspital, Department of Gynaecology and Gynaecological Oncology, Bern, Switzerland; ${ }^{2}$ Peter MacCallum Cancer Centre, Melbourne, Australia; ${ }^{3}$ University Hospital Bern, Inselspital, University of Bern, Department of Diagnostic, Interventional, and Pediatric Radiology, Bern, Switzerland

\subsection{6/ijgc-2021-ESGO.436}

Introduction/Background* Complete tumor resection (R0) at primary or interval debulking surgery is a main prognostic factor for overall survival in patients diagnosed with ovarian cancer. Neoadjuvant treatment has become standard of care in patients with advanced disease (FIGO stage IIIC/IV) or reduced performance status. Radiologic assessment of treatment response using CT scans has a low prediction for complete surgical tumor resection.

Here we aim to identify prognostic factors associated with R0-resection after neoadjuvant chemotherapy (NACT) for ovarian cancer and propose a multimodal scoring system using 3 Tesla diffusion-weighted MRI (DW-MRI), CA-125 and diagnostic laparoscopy for future investigation.

Methodology All patients treated with neoadjuvant chemotherapy for advanced primary ovarian, peritoneal or Fallopian tube cancer between 01/2012-12/2020 at the University Hospital Bern were included in this retrospective cohort study. Clinical and surgical data assessed include age, menopausal status,
ECOG performance status, radiologic findings, histologic subtype, FIGO stage, CA-125, Fagotti-score, surgical resection status and chemotherapeutic regimen.

Multiple MR-graphic findings are scored number and distribution of intra-abdominal and thoracal lesions, qualitative and quantitative diffusion restriction, lymph nodal (LN) status, as well as prevalence and size of cardio-phrenic LN. Following treatment, change in tumor and metastatic lesion size are assessed.

Result(s)* Overall, 130 out of 475 women with primary ovarian cancer treated at the University Hospital Bern between 2012 - 2020 underwent NACT. Mean age was 66.2 years (range 24-90). Most patients were diagnosed with high-grade serous subtype $(92 \%)$ at FIGO stage IIIC or IV (78\%). Interval debulking surgery was performed and resection status was noted for 112 patients after a mean of 3 neoadjuvant cycles. R0 resection was achieved in 80 patients (71.4\%), 10 patients $(8.9 \%)$ had residual disease $<1 \mathrm{~cm}$.

Until now, in our cohort, DW-MRI was performed in three patients during NACT.

Conclusion* We propose a multimodal scoring system for R0resection based on DW-MRI, CA-125 and Fagotti-Score assessed during diagnostic laparoscopy. To validate this score, a prospective multicentre study including women with suspected primary or recurrent ovarian cancer is planned.

\section{INFLUENCE OF SPLENECTOMY ON CHEMOTHERAPY TREATMENT AND ONCOLOGICAL PROGNOSIS IN WOMEN WITH ADVANCED OVARIAN CANCER}

${ }^{1} \mathrm{MT}$ Marina Martín*, ${ }^{1} \mathrm{~V}$ Lago, ${ }^{1} \mathrm{P}$ Padilla Iserte, ${ }^{2} \mathrm{AJ}$ Cañada, ${ }^{1} \mathrm{MT}$ Luis Javier, ${ }^{1} \mathrm{M}$ Gurrea, ${ }^{1} S$ Domingo. ${ }^{1}$ University Hospital La Fe, Gynecologic Oncology, Valencia, Spain; ${ }^{2}$ University Hospital La Fe, Biostatistics, Valencia, Spain

\subsection{6/ijgc-2021-ESGO.437}

Introduction/Background* To determine the effect of splenectomy on subsequent chemotherapy treatment and prognosis in women with advanced ovarian cancer.

Methodology We performed a retrospective study comparing two cohorts of patients. Data from 60 women who underwent splenectomy during cytoreductive surgery for primary or relapse ovarian cancer were compared with 62 controls who also underwent this type of surgery without splenectomy matched for baseline and surgical characteristics including type and date of surgery at University Hospital La Fe (Spain) between November 2011 and December 2019.

Result(s)* A total of $72 / 459(15,7 \%)$ women who underwent splenectomy for advanced ovarian cancer were identified. Twelve women were excluded and finally 60 cases and 62 controls were identified.

No differences were observed regarding the following variables: postoperative complications $(31.7 \%$ vs. $19.4 \%)$, mean time to start adjuvant chemotherapy (48.6 vs. 42.7 days), mean time to complete chemotherapy in women who received only adjuvant treatment (104 vs. 116 days) and percentage of six-cycle chemotherapy completion $(78.8 \%$ vs. $98.4 \%)$ after adjusting for a potential confounding factors. No differences were observed between groups related to cycles delayed $(50 \%$ vs. $32.3 \% ; \mathrm{P}=0.16)$ and reduction in the doses of chemotherapy $(23.3 \%$ vs. $22.6 ; \mathrm{P}=0,61)$; unlike the differences found according to cycles cancelled (30\% vs. $11.3 \% ; \mathrm{P}=0.037)$. Two women died in the splenectomy group (3.3\%). The mean 